\title{
Optical coherence tomography angiography in glaucoma: analysis of the vessel density-visual field sensitivity relationship
}

\author{
Gábor Holló \\ Department of Ophthalmology, Semmelweis University, Budapest, Hungary \\ Correspondence to: Gábor Holló. 1085. Budapest, Mária u. 39. Hungary. Email: hollo.gabor@med.semmelweis-univ.hu.
}

\begin{abstract}
Glaucoma, a well-defined group of progressive optic neuropathies is one of the leading causes of irreversible blindness worldwide. In order to stop or slow down the progression of glaucomatous vision deterioration, intraocular pressure reduction by medical, laser or surgical treatment is needed. To ensure that treatment is efficient and tailored to the actual needs both cross sectional evaluation of disease severity and measurement of rate of progression are essential. Currently staging and progression are investigated with visual field and retinal thickness measurements. Perimetry, however, is influenced by several biological factors which are not related to glaucoma, and the use of retinal thinning is limited by floor effect. Therefore, clinical application of optical retinal coherence tomography angiography, a new and rapidly developing noninvasive measurement of the capillary perfusion in the various retinal layers, respectively, is now in the focus of clinical glaucoma research. This comprehensive review summarizes the current knowledge on one of the most important research areas in optical coherence tomography angiography in glaucoma, the relationship between retinal capillary perfusion and the spatially corresponding visual field threshold sensitivity.
\end{abstract}

Keywords: Glaucoma; OCT vessel density; optical coherence tomography angiography; Octopus perimetry; visual field sensitivity; structure-function relationship

Submitted Jan 15, 2020. Accepted for publication Mar 13, 2020.

doi: 10.21037/atm.2020.03.187

View this article at: http://dx.doi.org/10.21037/atm.2020.03.187

\section{Pathophysiology of glaucoma}

Glaucoma is a group of progressive optic neuropathies, in which the pattern of retinal nerve fiber layer loss and the consequential progressive damage to the optic nerve head (disc) and visual field are characteristic; this separates glaucoma from the other optic neuropathies (1). The retinal nerve fiber layer (RNFL) comprises the axons of the retinal ganglion cells (RGCs). Fifty percent of the RGCs are located in the macular area in an area approximately $6 \mathrm{~mm}$ in diameter around the retinal location of the central sharp vision (fovea). In glaucoma the primary damage involves the retinal nerve fibers deep in the optic nerve head (in the lamina cribrosa), and the loss of the cell bodies (RGCs) is secondary. The clinical presentations of the various primary and secondary glaucoma types are diverse, but a common feature of their pathomechanisms is progressive damage of the retinal nerve fibers at the level of lamina cribrosa. In primary open-angle glaucoma (POAG), which is the most common form of glaucoma in Europe, USA and Africa, vascular dysregulation in the optic nerve head and the superficial peripapillary retina where the RNFL takes place, and in the superficial macular retina where the macular RGCs are located, is frequently part of the pathomechanism (2). Development and progression of the glaucomatous structural damage (optic nerve head cupping, RNFL and inner macular retina thinning due to RGC loss), and the resulting functional deterioration (visual field deterioration) are typically local changes, which combine in advanced glaucoma to constitute diffuse structural and functional damage. Thus, local (sector, area) measurements are important in glaucoma, and cannot be replaced by global structural and functional assessments. 


\section{Visual field testing in glaucoma}

For four decades automated threshold perimetry of the central 30-degree visual field has been used to determine and characterize the functional damage (location, severity and shape). In addition to the total central 30-degree visual field, both test point clusters spatially corresponding to well-defined areas of the optic nerve head and peripapillary RNFL sectors, and the central 10-degree visual field and its superior and inferior 180-degree hemifields are of great importance in glaucoma $(3,4)$. The central 10 -degree visual hemifields represent the functioning of the opposite macular retinal hemifields in $6 \mathrm{~mm}$ diameter areas, respectively. They are of particular importance for vision-related quality of life, and provide information on the damage to the superficial macular retina, where $50 \%$ of the RGCs are found.

The visual field sensitivity threshold is determined for each preset test point location under standardized perimetric conditions $(3,4)$. The testing strategies, the test durations and the measurement precision levels vary. In general, the threshold lies between the strongest not-recognized and the weakest recognized illuminating light intensity values projected by the perimentry instrument (threshold zone). It is arbitrarily set to the middle of the threshold zone. In the most precise test strategies, the precision of the determined sensitivity threshold is approximately $\pm 1.0 \mathrm{~dB}$. For some predefined visual field areas (e.g., the central 30-degree and the macular 10-degree fields) threshold sensitivity is automatically averaged and provided by the perimeters' software functions. For practical clinical purposes the test results are also expressed as defect (deviation) from the corresponding normal values. The defect values are corrected for age, and show the deviation from the center of the age-related normal threshold range. They are automatically averaged for the total tested visual field and various visual field clusters and areas, respectively. When a threshold value remains within the normal range the defect value is zero. Since the light sensitivity of the human eyes covers a very wide range, it is not practical to use linear scaling in perimetry. Therefore, all values are presented on logarithmic scales and expressed in $\mathrm{dB}$.

The use of perimetry in glaucoma care is essential. However, it is limited by patient cooperation problems, technical difficulties, time constraints and artifacts, and the visual field reflects the influence of all diseases of the total visual pathway between the retinal photoreceptors and the brain.

\section{Structural optical coherence tomography measurements in glaucoma}

In the last two decades, RNFL thickness and inner (superficial) macular thickness measurements with optical coherence tomography (OCT) became established measures of glaucomatous tissue loss $(5,6)$. OCT offers thickness measurements for each of the layers in the retina, separately, using only one image acquisition. Thus, the thickness of layers that comprise RNFL and macular RGCs, respectively, can be measured separately from that of the other layers that are not involved in the glaucomatous tissue loss. The OCT-derived thickness results are given on linear scales and expressed in $\mu \mathrm{m}$. They contain total and sector peripapillary RNFL thickness and total, hemifield and sector macular thickness parameters. None of the thickness parameters is age corrected. OCT imaging for glaucoma is fast, can be used for progression analysis and requires less patient cooperation than perimetry, but it is influenced by artifacts (e.g., vitreous opacities, cataract and floor effect in advanced glaucoma). The OCT thickness measurements are not directly applicable to the assessment of visual functions and vision-related quality of life.

\section{Anatomical background of structure-function investigations in glaucoma}

Setting exact point-by-point spatial correspondence between a retinal location and a visual field test point location is clinically not possible due to differences in eye size and magnification, overlap between neighboring receptor fields, and influence of patient positioning on the results. However, area/sector correspondence (the Garway-Heath map) is established and has been widely used in structure-function investigations (7). The relationship between the spatially corresponding thickness and visual field sensitivity or mean defect values has been investigated for glaucoma for decades $(5,6,8,9)$. Though it is established for the peripapillary RNFL sectors and the macular area, the optimal use of a linear and a logarithmic scale for correlation remains controversial. In advanced glaucoma floor effect is a considerable barrier against using a structural test $(6,10)$. On the other hand, thickness parameters are stable in the short-term, and (in contrast to the perfusion parameters) are not influenced by short-term physiological or pathophysiological regulation. 


\section{Optical coherence tomography angiography in glaucoma}

Optical coherence tomography angiography (OCT angiography) comprises several rapidly evolving instrument and software systems which all offer noninvasive clinical blood perfusion measurement of selected layers of the retina and the optic nerve head (11). In contrast to conventional fluorescein or indocyanine green angiography, in which blood plasma is stained, OCT angiography detects the moving red blood cells by detecting their movement. The most developed OCT angiography systems offer selective capillary perfusion measurement, thus the effect of the large retinal arteries and veins is removed from the result. This is important because in glaucoma capillary perfusion of the retinal layers involved in the glaucomatous process carries clinically useful information. Larger vessels are subjects to regulatory effects (e.g., blood pressure changes) that are not related to glaucoma. Therefore, their influence may hamper accurate measurement of glaucomatous capillary perfusion changes (12). In glaucoma the most commonly used OCT angiography parameter is vessel density. Vessel density is the $\%$ of the area represented by red blood cell movement within the studied area in a layer of interest $(12,13)$. Other, less commonly used parameters are vessel length density, defined as the total length of perfused vasculature per unit tissue area, and perfusion density, defined as the total area of perfused vasculature per unit tissue area $(12,14,15)$.

In all types of glaucoma, vessel density decreases as a consequence of the glaucomatous tissue loss. However, in POAG vessel density is also an indicator of vascular dysregulation which is part of the pathophysiology of this glaucoma type $(2,6)$. Therefore, it has high diagnostic accuracy for POAG, and the measured values decrease parallel with increasing disease severity $(16,17)$. Compared to the retinal thickness parameters vessel density is considerably less influenced by floor effect, thus vessel density remains a clinically useful measure of glaucoma and glaucomatous progression in late stages of the disease $(18,19)$. Currently no OCT angiography parameter is age corrected, and no normal database is available for clinical classification purposes. It is important to note that segmentation for the different retinal layers is different in the various OCT angiography instrument families. Therefore, despite the identical or similar terminology used by the various instrument manufacturers, the measured results are not interchangeable between the systems.

In advanced OCT angiography systems, it is possible to obtain spatially corresponding structural and perfusion images and measurements using only one image acquisition for the area of interest. This possibility considerably reduces measurement noise, provides identical segmentation for the thickness and perfusion measurements, and uses identical retinal areas for structural and vessel density measurements (20). This advanced technology is essential to accurately investigate the relationship of the spatially corresponding retinal capillary perfusion, retinal thickness and visual field threshold sensitivity values in glaucoma (20). Figure 1 and 2 show a peripapillary and a macular OCT angiography/ thickness image with the corresponding vessel density and thickness values, respectively, for a POAG eye.

Measurement precision of the current OCT angiography instruments is supported by built-in motion correction and eye tracking functions. However, the measurements are still influenced by other types of noise (e.g., noise due to cataract, vitreous floaters and poor fixation) and the actual intraocular pressure (high intraocular pressure lowers the actual vessel density values) $(21,22)$. Breath holding and time elapsed since a chronic smoker's last cigarette, however, have no influence on the measured vessel density $(23,24)$.

\section{Vessel density—visual field threshold sensitivity relationship in glaucoma}

Global and sector relationships between retinal thickness and visual field sensitivity or defect values have been investigated for decades with various thickness measurement technologies (e.g., scanning laser polarimetry, time domain, Fourier domain and swept source OCT systems). It is now established that significant (moderate to very strong) relationships exist between the total central 30-degree visual field sensitivity and mean defect, and the 360-degree peripapillary RNFL thickness; between the peripapillary RNFL sectors' thickness values and the corresponding visual field cluster mean sensitivity and defect values; and between the superficial macular thickness and macular sensitivity, respectively $(6,8,9,13,16,20)$. However, for OCT angiography, which is a new technology, the relationships are still under investigation (10,13-16,18,20,25-29). In POAG reduction of capillary perfusion is an indicator of vascular dysfunction. Therefore, in POAG OCT angiography parameters may potentially be earlier indicators or predictors of early visual field deterioration and progression than the corresponding retinal thickness parameters. This is why the investigation of the quantitative 

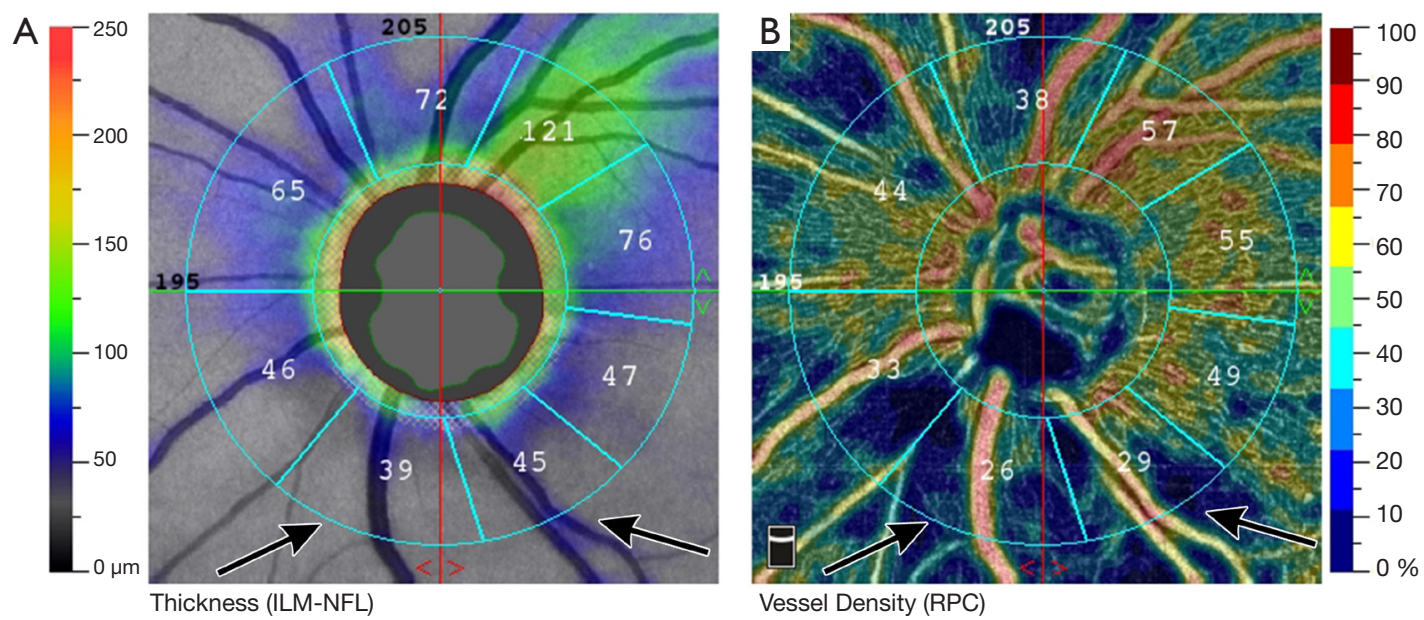

Figure 1 Optic nerve head and peripapillary optical coherence tomography angiography thickness/vessel density image of a primary openangle glaucoma eye (Angiovue OCT, Optovue Inc., Fremont, CA, USA). (A) Color-coded retinal nerve fiber layer thickness image with the peripapillary sectors and their thickness values expressed in $\mu \mathrm{m}$; (B) the corresponding vessel density image with the peripapillary sectors and their vessel density values expressed in \%. The localized vessel density/retinal nerve fiber layer defect is indicated with bluish color (arrows) and low thickness and vessel density values.
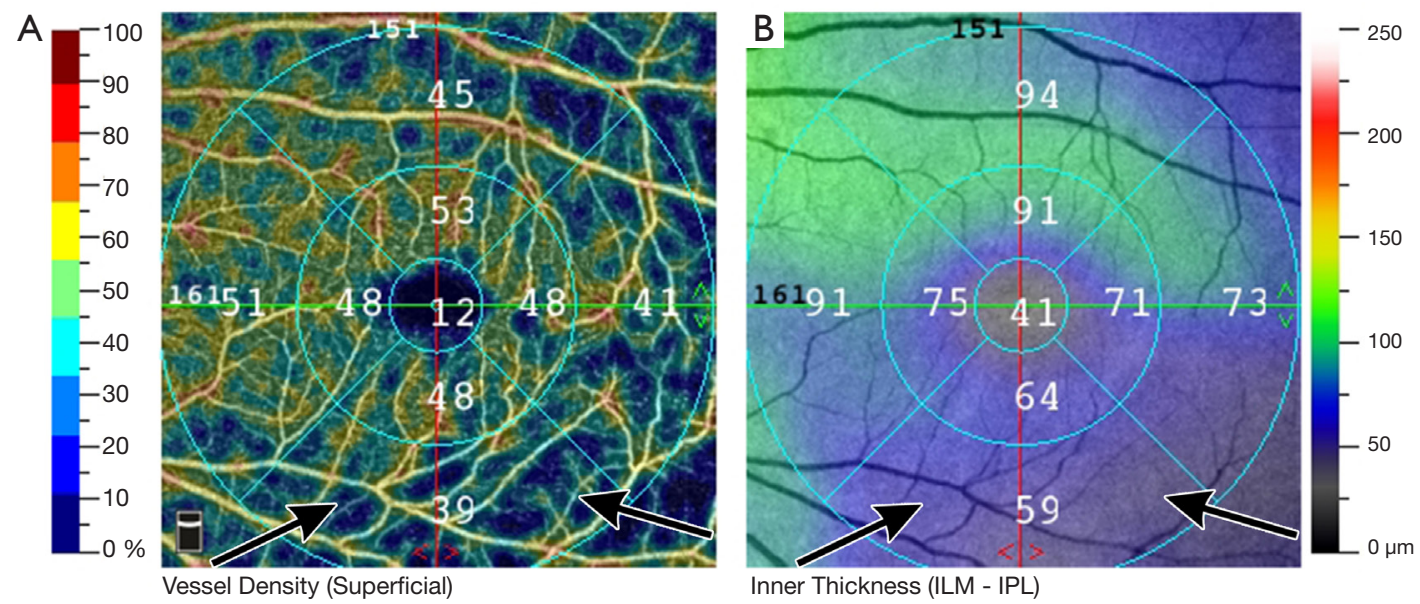

Figure 2 Macular optical coherence tomography angiography vessel density/thickness image (6-mm scan) of a primary open-angle glaucoma eye (Angiovue OCT, Optovue Inc., Fremont, CA, USA). (A) Color-coded superficial macular vessel density image with the macular sectors and their vessel density values expressed in \%; (B) the corresponding inner macular thickness image with the macular sectors and their thickness values expressed in $\mu \mathrm{m}$. The localized vessel density/retinal ganglion cell thickness defect is indicated with bluish color (arrows) and low vessel density and thickness values.

relationship between OCT angiography vessel density and visual field sensitivity is of great interest.

In order to make the vessel density-retinal sensitivity relationship applicable for clinical purposes, the relationship needs to be mathematically investigated. Since both linear and curvilinear relationships occur (depending on the transformation used, retina location investigated and subject/patient population studied) both linear correlation coefficients and linear and polinomial determination coefficients were calculated (10,13-16,18,20,25-29). No general best-performing analysis was found.

Regarding visual field related information, clinicians 
may use either exact threshold sensitivity values (the representative mean value calculated for the area of interest) or the respective defect values. In contrast to the sensitivity threshold parameters which are not provided automatically for the visual field clusters, the global and cluster mean defect values are available on the visual field reports $(3,8,13,16)$. This makes it easier for clinicians to use defect values for the vessel density-function calculations. In glaucoma, using visual field defect values revealed moderate to very strong relationships between the papillomacular, superotemporal and inferotemporal sector vessel density values, and the corresponding visual field areas, respectively $(13,15,16,18,26)$. However, this approach is not ideal since the defect values are age-corrected while the vessel density values are not, and the defect values reflect the difference between an age-corrected ideal normal value and the actual measured sensitivity value. This means that small to moderate sensitivity differences (depending on the visual field test strategy) are not reflected by the defect values, which may negatively influence the relationship in early glaucoma. Therefore, using age-uncorrected, individual test point threshold sensitivity values for the calculation of the area values offers a more precise basis for the investigation of vessel density (vessel length density, perfusion density) function relationship $(10,14,20,25-29)$.

Similarly to the retinal thickness parameters, vessel density is measured on a linear scale, while retinal sensitivity is given on a logarithmic scale. This represents a problem for correlation studies since, similarly to thickness-function correlations, plotting a linear and a logarithmic parameter scale may result in a curvilinear relationship. Theoretically there are four possible ways to present data for vessel density-retinal sensitivity correlations. In the first, each of the individual test point sensitivity values within the visual field area of interest is converted to a linear $(1 / \mathrm{L})$ scale using the $\mathrm{dB}=10 \times \log 10(1 / \mathrm{L})$ formula (unlogged values). Then the unlogged threshold sensitivity values are averaged, and the mean value is correlated with the corresponding vessel density parameter. This is the most commonly applied approach $(14,15,20,25,27-29)$. The problem with this widely used technique is that if the studied visual field area contains one or more points with high (preserved) sensitivity and other points with reduced sensitivity the unlogged average value mainly reflects the points with high threshold sensitivity. Thus, visual field deterioration may be underrepresented. The second possible approach involves converting the sector vessel density (vessel length density, perfusion density) value from linear to logarithmic scale.
The third option is to average the threshold sensitivity values in $\mathrm{dB}$, and then unlog the mean $\mathrm{dB}$ value. Finally, one can simply correlate the vessel density values measured on a linear scale and the averaged visual field threshold sensitivity values measured on a logarithmic scale. Using the data of a recent original study published by us (20) we graphically present three different approaches in Figure 3 and the corresponding correlation coefficients in Table 1. In the original study the means of unlogged threshold sensitivity values of the superior and inferior 10-degree visual hemifields were correlated with the spatially corresponding (opposite) macular retina hemifield vessel density values in an area $6 \mathrm{~mm}$ in diameter, respectively. In Figure 3 inferior macular vessel density is plotted against superior macular hemifield threshold sensitivity for the total study population, which comprised 27 POAG eyes and 23 non-glaucomatous eyes. The curves and relationships were similar for the opposite hemifield, and for both hemifields when the POAG eyes, and the non-glaucomatous eyes were evaluated separately (plots are not shown). The abscissa axis is vessel density in \% (linear scale). In Figure $3 A$ the ordinate axis is linear (average of the unlogged sensitivity values). In Figure $3 B$ it is logarithmic (average of the original sensitivity values in $\mathrm{dB}$ ). In Figure $3 C$ it is once again linear (unlogged average of the original sensitivity values). Figure $3 B$ shows that the linear $v s$. logarithmic plotting results in a curvilinear relationship, thus using linear correlation is theoretically not optimal. But the correlations are all very strong, similar, and statistically significant, and there is no systematically best-performing correlation approach (Table 1). This suggests that the type of data transformation had limited impact on the correlation strength. Thus, no rigorous transformation criteria can be recommended for vessel density-visual function investigations.

In recent years correlation studies have shown that in POAG the spatially corresponding thickness and vessel density values measured in the peripapillary retinal sectors and the macula both show significant relationships with the corresponding visual field sensitivity/defect values. However, irrespective of which perimetry system and test strategy were employed, the relationship was consistently stronger for vessel density in those retinal areas which typically display early damage in POAG: the inferotemporal peripapillary area and the macular hemifields $(16,18,20,24,25,29)$. These results suggest that further detailed analysis of the correlation between vessel density and visual field sensitivity for small but clinically important retinal areas is needed to improve our understanding of the 

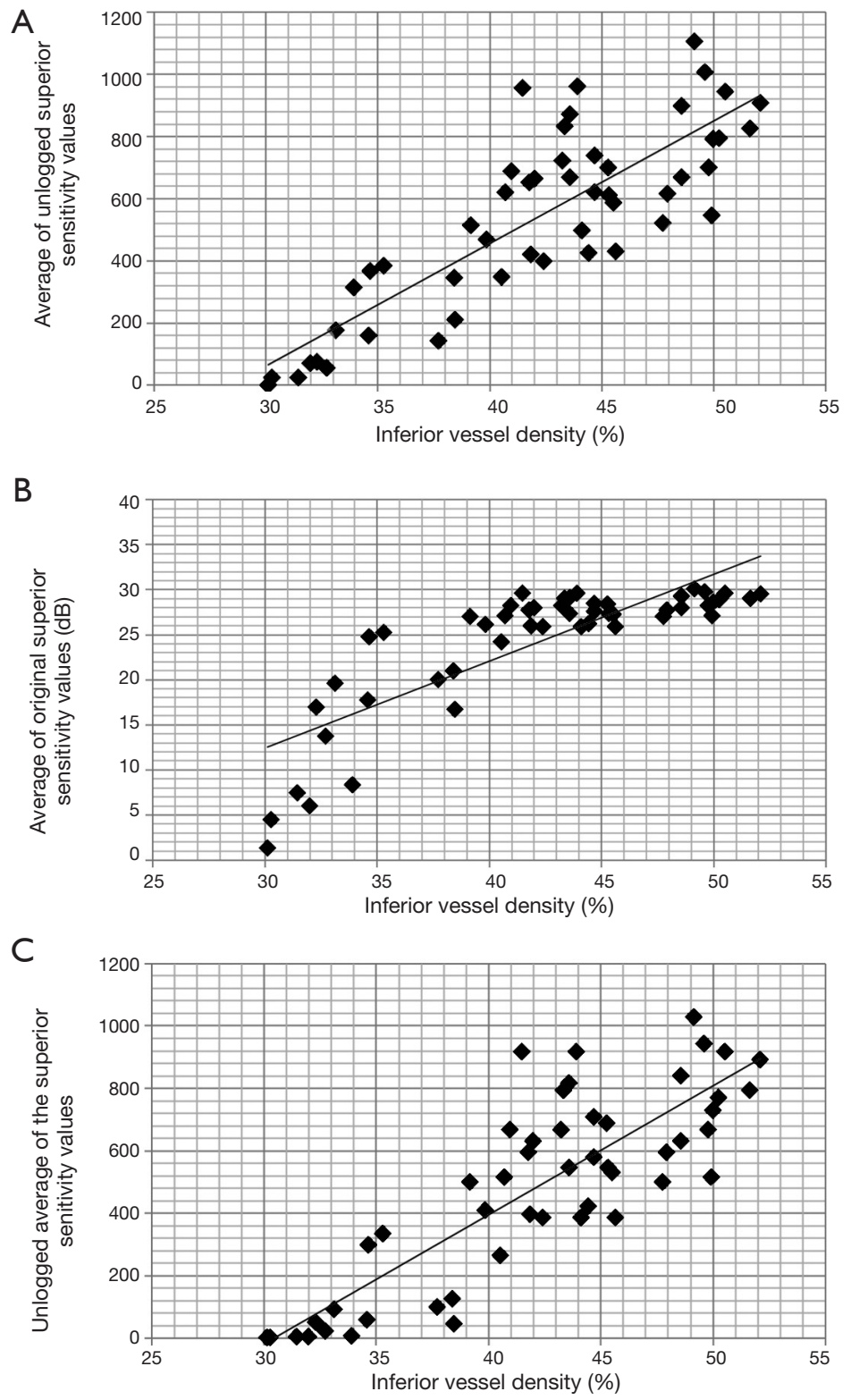

Figure 3 Correlation between the spatially corresponding inferior macular hemifield vessel density expressed in \% and superior macular visual hemifield sensitivity calculated in three different ways for a group comprising 23 non-glaucomatous eyes and 27 primary open-angle glaucoma eyes. (A) threshold sensitivity is expressed as the average of the unlogged individual threshold sensitivity values (linear scale); (B) threshold sensitivity is expressed as the average of the original threshold sensitivity values in db (logarithmic scale); (C) threshold sensitivity is expressed as the unlogged average of the original threshold sensitivity values (linear scale).

development and progression of glaucomatous functional deterioration and its progression in POAG.

\section{Technical limitations in vessel density-visual field sensitivity correlation studies}

Correlation studies that investigate the relationship between
OCT angiography and visual field sensitivity parameters are considerably influenced by OCT angiography image quality, pathophysiological factors that modify retinal capillary perfusion, and the technical characteristics of the perimetry system/test/strategy used in the research. The OCT angiography images must undergo both software-provided quality checking (image quality scoring) and a subjective 


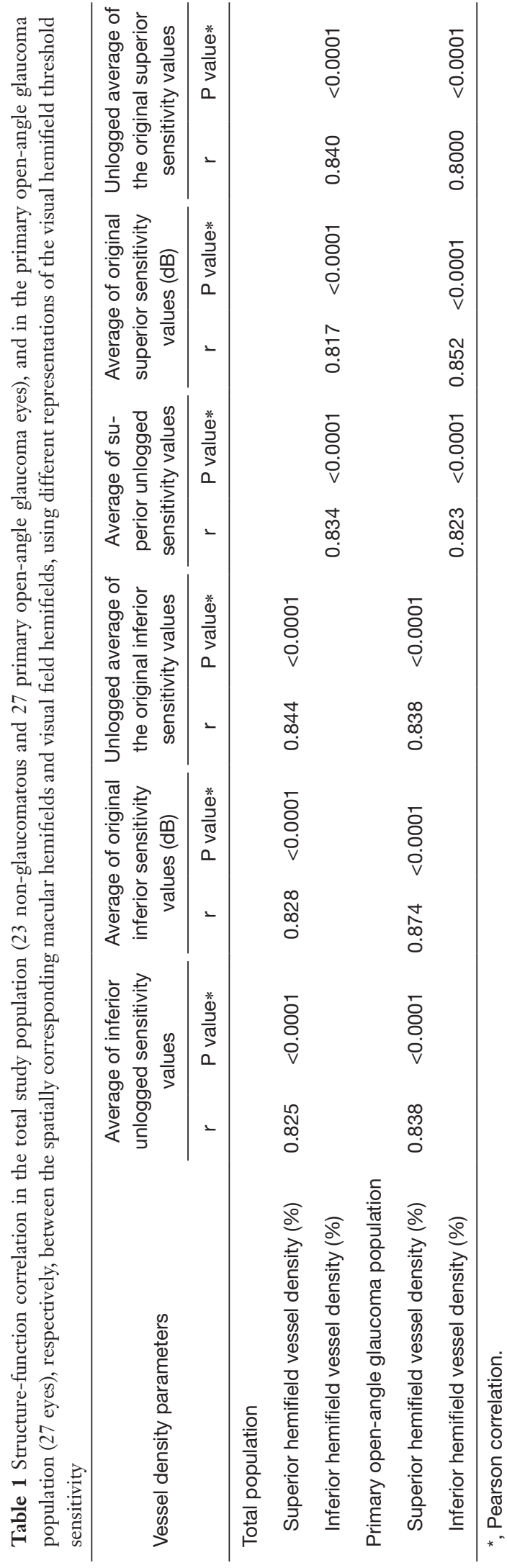

evaluation by the operator for segmentation errors, shadows, and artifacts caused by eye movements, cataract, vitreous floaters and vitreoretinal abnormalities. The quality requirements are much higher for quantitative research than for routine clinical use. Eyes with other diseases (e.g., diabetic retinopathy) are not suited to glaucoma correlation studies since vessel density is influenced by the other coexisting diseases. When blood pressure or intraocular pressure is not controlled during image acquisition the OCT angiography result can differ considerably from that acquired under controlled conditions (26). Since in clinical practice many glaucoma patients show one or more of the above-mentioned conditions the number of patients eligible to prospective correlation studies is limited.

Of the various clinically used perimetry systems the Octopus perimeter family (3) and the Humphrey Visual Field Analyzer family (4) are established for vessel densityvisual field correlation studies $(10,14,20,25-29)$. These advanced systems, however, differ essentially from each other in their test point distribution, particularly within the central visual field area $(3,4,30)$, which may result in different correlation strengths.

The above-mentioned considerations show that high quality capillary perfusion-visual field sensitivity correlation studies are difficult to conduct. Medical records retrospectively selected from the routine glaucoma practice may not meet the above quality criteria. Therefore, they may not help to clarify fine or localized relationships.

\section{Potential clinical application of vessel density- retinal threshold sensitivity investigations}

Glaucoma is a progressive optic neuropathy, thus, it requires long-term management. Detection of early progression is important as it enables clinicians to introduce treatment intensification in a timely manner. Currently, detection of glaucoma progression is based on visual field and structural OCT progression analysis by the linear regression analysis software functions of the testing instruments. However, both analyses are negatively influenced by long-tem variability and insufficient test quality/reliability. The retinal thickness parameters reflect established RNFL loss and RGC death. By the time their progression becomes statistically significant glaucomatous tissue damage will already have occurred. In contrast, in POAG capillary perfusion changes are potential early indicators of the dysfunction of not-yet-lost RNFL and RGCs. This is why OCT angiography perfusion-visual field sensitivity 
correlations may be of value in glaucoma care. Investigation of the relationship between vessel density change and corresponding visual field sensitivity change may help to clarify the potential role of OCT angiography in the detection of early glaucomatous dysfunction, prior to the development of irreversible tissue loss.

\section{Conclusions}

Investigation of the relationship between global, peripapillary sector, and macular hemifield OCT angiography vessel density and the spatially corresponding visual field area sensitivity, respectively, is one of the main research fields in OCT angiography in glaucoma. The published investigations have established strong and statistically significant relationships. In POAG, in retinal areas that show early damage the correlations are stronger than those found for the spatially corresponding thickness parameters. Future investigations are needed to specify measurement and calculation methods for long-term studies on the correlation of OCT angiography change and visual field sensitivity change in glaucoma.

\section{Acknowledgments}

Funding: None.

\section{Footnote}

Provenance and Peer Review: This article was commissioned by the editorial office, Annals of Translational Medicine for the series "OCT Angiography in Glaucoma". The article was sent for external peer review.

Conflicts of Interest: The author has completed the ICMJE uniform disclosure form (available at http://dx.doi. org/10.21037/atm.2020.03.187). The series "OCT Angiography in Glaucoma" was commissioned by the editorial office without any funding or sponsorship. GH served as the unpaid Guest Editor of the series and serves as an unpaid editorial board member of Annals of Translational Medicine from Dec 2019 to Nov 2021. The author reports non-financial support from Optovue ltd, during the conduct of the study.

Ethical Statement: The author is accountable for all aspects of the work in ensuring that questions related to the accuracy or integrity of any part of the work are appropriately investigated and resolved.

Open Access Statement: This is an Open Access article distributed in accordance with the Creative Commons Attribution-NonCommercial-NoDerivs 4.0 International License (CC BY-NC-ND 4.0), which permits the noncommercial replication and distribution of the article with the strict proviso that no changes or edits are made and the original work is properly cited (including links to both the formal publication through the relevant DOI and the license). See: https://creativecommons.org/licenses/by-nc$\mathrm{nd} / 4.0 \%$.

\section{References}

1. European Glaucoma Society. Terminology and Guidelines for Glaucoma. 4th ed. Savona: PubliComm, 2014.

2. Kwon J, Jo YH, Jeong D, et al. Baseline systolic versus diastolic blood pressure dip and subsequent visual field progression in normal-tension glaucoma. Ophthalmology 2019;126:967-79.

3. Racette L, Fischer M, Bebie H, et al. Visual field digest: A guide to perimetry and the Octopus perimeter. 7th ed. Köniz: Hag-Streit AG, 2017.

4. Humphrey Field Analyzer II- series, User Manual. Dublin: Carl Zeiss Meditec, Inc., 2010.

5. Torres LA, Hatanaka M. Correlating structural and functional damage in glaucoma. J Glaucoma 2019;28:1079-85.

6. Sung MS, Heo H, Park SW. Structure-function relationship in advanced glaucoma after reaching the RNFL floor. J Glaucoma 2019;28:1006-11.

7. Garway-Heath DF, Poinoosawmy D, Fitzke FW, et al. Mapping the visual field to the optic disc in normal tension glaucoma eyes. Ophthalmology 2000;107:1809-15.

8. Holló G. Comparison of structure-function relationship between corresponding retinal nerve fibre layer thickness and Octopus visual field cluster defect values determined by normal and tendency-oriented strategies. $\mathrm{Br} \mathrm{J}$ Ophthalmol 2017;101:150-4.

9. Tan O, Greenfield DS, Francis BA, et al. Estimating visual field mean deviation using optical coherence tomographic nerve fiber layer measurements in glaucoma patients. Sci Rep 2019;9:18528.

10. Kim G-N, Lee EJ, Kim H, KIm T-W. Dynamic range of the peripapillary retinal vessel density for detecting glaucomatous visual field damage. Ophthalmology Glaucoma 2019;2:103-10. 
11. Tan ACS, Tan GS, Denniston AK, at al. An overview of the clinical applications of optical coherence tomography angiography. Eye (Lond) 2018;32:262-86.

12. Holló G. Influence of removing the large retinal vesselsrelated effect on peripapillary vessel density progression analysis in glaucoma. J Glaucoma 2018;27:e137-9.

13. Holló G. Relationship between OCT angiography temporal peripapillary vessel-density and Octopus perimeter paracentral cluster mean defect. J Glaucoma 2017;26:397-402.

14. Rao HL, Riyazuddin M, Dasari S, et al. Relationship of macular thickness and function to optical microangiography measurements in glaucoma. J Glaucoma 2018;27:210-8.

15. Rao HL, Dasari S, Riyazuddin M, et al. Diagnostic ability and structure-function relationship of peripapillary optical microangiography measurements in glaucoma. J Glaucoma 2018;27:219-26.

16. Holló G. Relationship between optical coherence tomography sector peripapillary angioflow-density and Octopus visual field cluster mean defect values. PLoS One 2017;12:e0171541.

17. Yarmohammadi A, Zangwill LM, Diniz-Filho A, et al. Optical coherence tomography angiography vessel density in healthy, glaucoma suspect, and glaucoma eyes. Invest Ophthalmol Vis Sci 2016;57:OCT451-9.

18. Lee SH, Lee EJ, Kim TW. Comparison of vascularfunction and structure-function correlations in glaucomatous eyes with high myopia. Br J Ophthalmol 2020;104:807-12.

19. Moghimi S, Bowd C, Zangwill LM, et al. Measurement floors and dynamic ranges of OCT and OCT angiography in glaucoma. Ophthalmology 2019;126:980-8.

20. Holló G. Comparison of thickness - function and vessel density - function relationship in the superior and inferior macula, and the superotemporal and inferotemporal peripapillary sectors. J Glaucoma 2020;29:168-74.

Cite this article as: Holló G. Optical coherence tomography angiography in glaucoma: analysis of the vessel density-visual field sensitivity relationship. Ann Transl Med 2020;8(18):1203. doi: 10.21037/atm.2020.03.187
21. Holló G. Influence of posterior subcapsular cataract on structural OCT and OCT angiography vessel density measurements in the peripapillary retina. J Glaucoma 2019;28:e61-3.

22. Holló G. Influence of large intraocular pressure reduction on peripapillary OCT vessel density in ocular hypertensive and glaucoma eyes. J Glaucoma 2017;26:e7-10.

23. Holló G. Valsalva maneuver and peripapillary OCT angiography vessel density. J Glaucoma 2018;27:e133-6.

24. Holló G. No acute effect of smoking on peripapillary and macular vessel density in healthy middle-aged smokers. J Glaucoma 2019;28:e86-8.

25. Shin JW, Kwon J, Lee J, et al. Relationship between vessel density and visual field sensitivity in glaucomatous eyes with high myopia. Br J Ophthalmol 2018. [Epub ahead of print].

26. Sakaguchi K, Higashide T, Udagawa S, et al. Comparison of sectoral structure-function relationships in glaucoma: vessel density versus thickness in the peripapillary retinal nerve fiber layer. Invest Ophthalmol Vis Sci 2017;58:5251-62.

27. Penteado RC, Zangwill LM, Daga FB, et al. Optical coherence tomography angiography macular vascular density measurements and the central 10-2 visual field in glaucoma. J Glaucoma 2018;27:481-9.

28. Shin JW, Lee J, Kwon J, et al. Regional vascular densityvisual field sensitivity relationship in glaucoma according to disease severity. Br J Ophthalmol 2017;101:1666-72.

29. Shin JW, Lee J, Kwon J, et al. Relationship between macular vessel density and central visual field sensitivity at different glaucoma stages. Br J Ophthalmol 2019;103:1827-33.

30. Roberti G, Manni G, Riva I, et al. Detection of central visual field defects in early glaucomatous eyes: Comparison of Humphrey and Octopus perimetry. PLoS One 2017;12:e0186793. 